\title{
A life dedicated to informatics: an obituary for Prof. Friedrich L. Bauer
}

\section{Manfred Broy}

Published online: 23 May 2015

(C) Springer-Verlag Berlin Heidelberg 2015

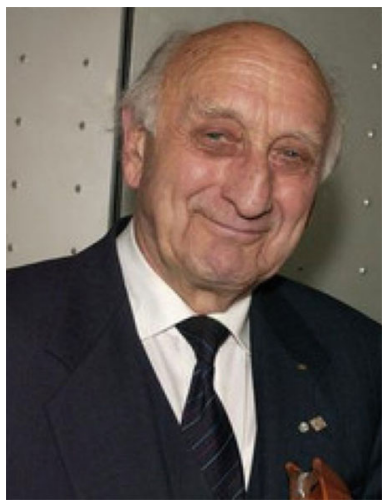

On Thursday, the 26th April 2015, Friedrich L. Bauer, Professor emeritus at the Faculty of Informatics at the Technischen Universität München, passed away at the age of 90 following a prolonged illness. Informatics in Germany looses one of its most influential pioneers. With his contributions, Friedrich L. Bauer decisively influenced over several stages the development of a discipline that shaped our world like no other in the last 50 years.

After WW2 and a short period of being a prisoner of war, Friedrich Bauer started his studies in mathematics and physics. He worked towards his Ph.D. under the supervision Prof. Fritz Bopp, the successor of Arnold Sommerfeld at the chair of Theoretical Physics at the Ludwig-Maximilians-Universität München. Already after 3 years, he obtained his doctoral degree. Inspired by a report of the Swiss professor Heinz Rutishauser on the topic of electrical computers, Bauer studied Claude Shannon's switching algebra as well as the work of John von Neumann. Bauer's attention was drawn to a confidential seminar of mathematician Robert Sauer, professor for Mathematics for Engineers at the Technischen Universität München, 
and Hans Piloty, professor for Telecommunications. Their goal was the development of an electronic computer, which later became well-known under the name of PERM. In collaboration with his friend and congenial fellow student Klaus Samelson and Heinz Schecher, a physicist, Bauer developed the floating-point arithmetic of the PERM as well as the corresponding micro codes. Schecher discovered the crucial concept of indirect addressing and automatic address substitution.

1956 PERM is operational. Despite this triumph, the creators had to find that the hardware, based on electronic tubes, was disagreeably fragile. In addition, their programs proved error prone. Bauer related programming the PERM to the laborious process of embroidery. These experiences lead him to a topic that he pursues for the rest of his life: the quest for adequate programming languages and methods. With Klaus Samelson, he worked on a solution to the dilemma of excessively complicated programming. Programming languages are a potential solution. However, how should machines be able to process languages that differ widely in their sets of operations? The answer was evident: Compilers, "programming programs", as Bauer's friend Andrej Ershov of the former UdSSR used to call them-programs translating other programs to machine code.

Translation is centred on the stack method. The stack method saves intermediate results from the evaluation of complex expressions in a "last in-first out" fashion: new results are "pushed" on the stack and "popped" off when needed, with the most recent item being the first to be removed. Bauer and Samelson filed a patent for the stack method in 1957. Next they looked for a programming language; however, FORTRAN was not a desired choice. Programming languages originating from the US were excessively dominated by the overpowering US industry. However, a programming language requires frequent use and frequent implementations if it is to achieve its full potential. An international committee designed the programming language ALGOL 60, arguably the most important and prominent milestone in the history of programming languages. ALGOL 68 and $\mathrm{C}$ are direct successors of ALGOL 60. Also the object-oriented language SIMULA 67, the mother of all object-oriented languages, such as Java, and PASCAL are successors of ALGOL 60.

Transcending his work on programming languages, Bauer-at this time already holding a chair at the Technische Universität München-recognized that programming is more than a craft and he began a quest for its scientific core. Despite his continuous work in numerics, he turned his attention to building up the discipline of informatics, thus following the goal of Sauer to form information science as a discipline in its own right.

With dedication and persistence, Bauer pursued the foundation of the commission for informatics and the enlargement of the data center of the Bavarian Academy of Science, the Leibniz Rechenzentrum (LRZ). Progress also occured on the national level-the ministry of science in Bonn founded an advisory board for the development of a university curriculum for information science. To study the current state of the art, Bauer traveled to the US. The German "Rektorenkonferenz" founded an expert committee and, in 1969, started a national research project with a budget of four million Deutsch Mark. Rarely, such an important sum of money has been this well invested.

In 1969, Bauer initiated the founding of the Gesellschaft für Informatik (German Informatics Society), obtaining member number 1. At this point, the term of "informatics" was already widely used, a new and programmatic word, superior to "Computer Science", as commonly used in English, or the only marginally better "Computing Science"-with the systematic and specific processing of information being at the heart of the matter, not computers or "computing". From his first lectures in informatics, Bauer avoided to restrict the focus of the discipline to programming techniques. He defined informatics as scientific discipline of the representation, storage, processing, and transmission of information and stresses the 
historical roots as well as the scientific dimensions of informatics. Two seminal introductory books further amplified Bauer's determining influence.

Towards the end of the 1960s, it became apparent that despite the use of the new programming languages, the development of large software systems exceeded the capabilities of programmers. Bauer recognized early on that neither programming language nor the action of programming per se pose the main challenge in the development of large and complex software systems. Bauer was responsible for the organization of a congress in GarmischPartenkirchen-nowadays considered legendary, of historic importance. In the course of this event, Bauer coined the term "Software Engineering", a programmatic guide for the development of informatics as an engineering discipline.

Bauer dedicated himself to discover the scientific basis for the development of large software systems, for instance, in the project CIP with the title "Computer-aided, Intuitionguided Programming" that is still of meaning today. The outcome is documented in the book "Algorithmic Language and Program Development" which unites algebra, logic, and informatics to form a solid foundation for the development of software systems.

From the late 1970s, Bauer turns to his early and late scientific passion, cryptology and cryptography. Thus, he early on paved the way for a topic that nowadays dominates the discourse of digitalization: information security. After retiring, he dedicated himself to the historical roots of informatics more than anyone else. His contributions to the development of the department of informatics at the Deutsches Museum in Munich are invaluable. In his recently published "Short History of Informatics", Bauer describes the early contributions to the field from Al Chwarizmi, Ramon Lull and Gottfried Wilhelm Leibniz to Konrad Zuse, Alan Turing, Norbert Wiener, Edsger W. Dijkstra and Niklaus Wirth.

For his seminal work, Professor Bauer received numerous honors-amongst others the Computer Pioneer Award of the IEEE Computer Society and the membership at the German Academy of Science Leopoldina and the Bavarian Academy of Science. In his personal life, as a lovingly cared for husband, father of five, and grandfather to numerous grandchildren, his heart belonged to his family.

The triumph of informatics will continue. Many scientific, technical, societal, and economical challenges remain to be addressed. Professor F. L. Bauer's fundamental contributions to the disciplin, as well as the determination of his biography, have given direction and foundation to informatics in Germany. F. L. Bauer passionately united the practical relevance of informatics and its important role in the creation of large software systems with a rigorous scientific foundation in research and teaching. This stance also determined his dedication for the historical dimension of informatics, a topic whose relevance he continuously pronounced and researched.

With the passing of F. L. Bauer, we lost our founder, our oldest and most significant member of the board of Acta Informatica, who contributed to the success and the development of the journal in many respects. We will sadly miss him. 